\title{
Surfactant Protein-C in the Maintenance of Lung Integrity and Function
}

\section{Stephan W. Glasser ${ }^{1 *}$, John E. Baatz ${ }^{2}$ and Thomas R. Korfhagen ${ }^{1}$}

${ }^{1}$ Department of Pediatrics, Cincinnati Children's Hospital Medical Center, University of Cincinnati College of Medicine, Cincinnati, Ohio, USA

${ }^{2}$ Department of Pediatrics, Medical University of South Carolina and MUSC Children's Hospital; Charleston, South Carolina, USA

\begin{abstract}
Surfactant protein-C (SP-C) is a lung cell specific protein whose expression is identified from the earliest stages of mammalian lung development in a subset of developing epithelial cells and in the alveolar type II cell in the mature lung. Although SP-C gene expression is not critical and protein function is not necessary for the normal developing morphological patterning of the lung, studies of SP-C protein mutations and SP-C deficiency have revealed critical roles of SP-C in the maintenance and function of the preterm and mature lung during various forms of intrinsic or extrinsic lung injury. This review summarizes studies using in vitro experimental approaches, in vivo modeling in transgenic mice, and analysis of human disease pathogenesis. Collected data reveal an essential role for SP-C singly and in combination with other lung proteins, in maintenance of lung structure and pulmonary function of the immature and mature lung.
\end{abstract}

\section{Introduction}

Development of the lung begins during mid embryogenesis as an outgrowth of the foregut endoderm. Establishment of the respiratory tree involves the progressive growth and branching of endodermal tissue that invades pulmonary mesenchyme to produce an extensive conducting network that terminates in saccules. These saccular structures in turn must dilate and undergo septal extension, thinning, and division to form the acinar units that mature into efficient gas exchanging alveoli. Extension by the invading epithelium requires the simultaneous growth in parallel of the pulmonary vasculature to produce a fine lattice-like capillary bed that surrounds the developing alveolar structures. Lung development and alveologenesis does not terminate at birth. While initial alveolar maturation occurs prenatally in humans the expansion of the number of alveoli continues well into postnatal life [1]. Hypoxia and childhood illnesses influence the final number of alveoli produced [2]. Postnatal lung development is more extensive in mice than in humans. Alveolar units continue to be added after birth and the differentiation of the alveolar epithelium occurs perinatally in mice. Evaluation of term and postnatal septation in rodents indicates that two thirds of alveoli are formed after birth [3]. The mammalian lung is exposed to a variety of injurious substances. Both the developing lung in preterm infants and the mature lung can be injured by infection or inhalation of environmental toxicants which can result in aberrant lung remodeling and function leading to acute or chronic lung diseases. Studies of innate inmmune molecules in the lung show that many of these molecules including SP-C are produced in the prenatal developing lung.

In the mature lung, the proximal airway to distal alveolar surfaces of the lung are lined by a continuum of highly differentiated epithelial cell types. These cells synthesize and secrete material to preserve hydration, protect against inspired irritants or pathogens and maintain lung compliance. Pulmonary mucin imparts protective functions along the conducting surface of airways while pulmonary surfactant shields the alveolar surface and portions of the conducting airway. Pulmonary surfactant is the critical protective phospholipid - protein complex that reduces surface tension and prevents alveolar collapse at end expiration. Surfactant deficiency in the preterm infant is a major cause of neonatal respiratory distress syndrome (RDS) [4,5]. In full term infants impaired surfactant metabolism and production also cause acute respiratory failure. Important among the goals of pulmonary research has been the identification of critical genes and networks regulating lung development, function, and recovery from injury. These studies of the developing lung have led to identification of surfactant proteins which although produced in the developing lung also have roles in the maintenance of the mature lung. Such studies have been important in identifying necessary surfactant proteins in bovine and porcine surfactant extracts that are used in treating respiratory distress in infants.

Surfactant proteins (SP-A, SP-B, SP-C and SP-D) facilitate surface absorption and spreading of the surfactant phospholipid film or bind to microbial pathogens to enhance their clearance and prevent or reduce the severity of infection. However, all four of the surfactant proteins have distinctive structures, functional properties, and patterns of expression. SP-A and SP-D are aqueous soluble proteins that harbor collagen like domains and lectin-like binding domains and are thus commonly referred to as collectins. The collagen sequences form higher ordered oligomers while the lectin binding motifs mediate microbial binding and aggregation [6]. SP-B and SP-C are highly hydrophobic peptides that associate with the surfactant phospholipids. SP-B has multiple amphipathic helical domains and is essential for intra-cellular surfactant formation and extracellular surface activity [7]. SP-C is the most hydrophobic of the peptides and only 35 amino acids in length $[4,8]$. Cloned segments of the SP-C gene promoter have been used as probes of alveolar epithelial cell development and to identify transcription factors that determine cell specific expression. These SP-C promoter elements are widely used to alter lung development and model lung disease in transgenic mice [9]. Defects of SP-C expression underlie some forms of human lung disease and mutated or deficient SP-C cause interstitial lung disease (ILD) and idiopathic pulmonary

*Corresponding author: Stephan W. Glasser, PhD, Division of Neonatology, Perinatal and Pulmonary Biology, The Perinatal Institute, Department of Pediatrics MLC 7029, Cincinnati Children's Hospital Medical Center, 3333 Burnet Ave, Cincinnati, Ohio, USA 45229-3039, E-mail: steve.glasser@cchmc.org

Received November 23, 2011; Accepted December 17, 2011; Published December 21, 2011

Citation: Glasser SW, Baatz JE, Korfhagen TR (2011) Surfactant Protein-C in the Maintenance of Lung Integrity and Function. J Aller Ther S7:001. doi:10.4172/21556121.S7-001

Copyright: (c) 2011 Glasser SW, et al. This is an open-access article distributed under the terms of the Creative Commons Attribution License, which permits unrestricted use, distribution, and reproduction in any medium, provided the original author and source are credited. 
Citation: Glasser SW, Baatz JE, Korfhagen TR (2011) Surfactant Protein-C in the Maintenance of Lung Integrity and Function. J Aller Ther S7:001. doi:10.4172/2155-6121.S7-001

fibrosis (IPF). The role of SP-C in human lung development, disease and application of the SP-C promoter to model injury is the subject of this review.

\section{Control of Surfactant Synthesis and Catabolism is Essential for Respiratory Function and Alveolar Stasis}

The production of pulmonary surfactant is a highly structured

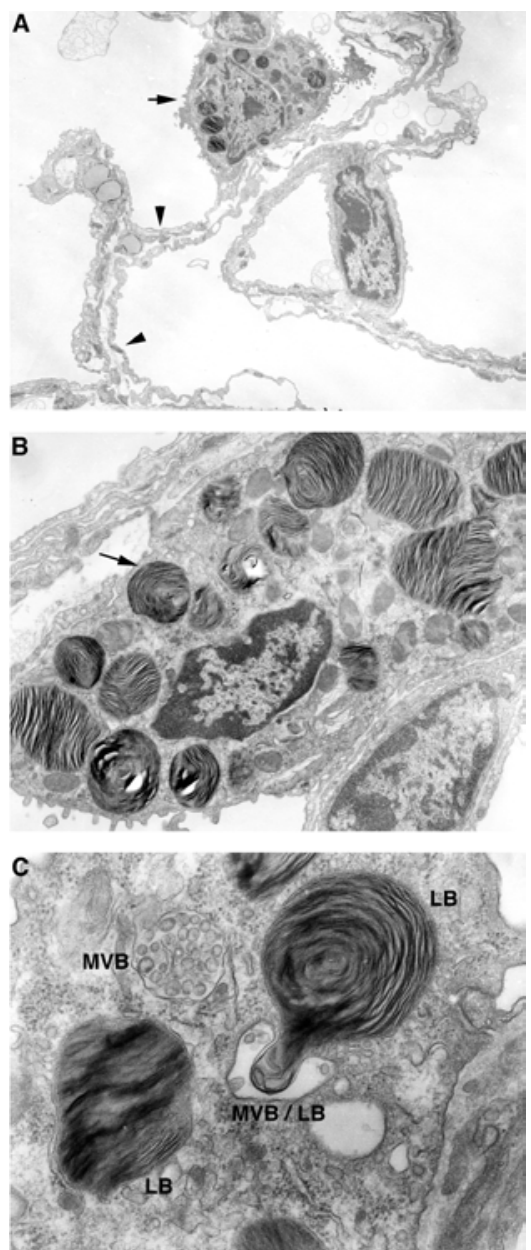

Figure 1: Ultrastructural detail of alveolus, type II cell and surfactant biosynthetic elements

A: The alveolar surface is line by an epithelium of two distinct differentiated cell populations. A small set of cuboidal type II epithelial cells (arrow) produce and secrete surfactant onto the alveolar lumen, note surface microvilli and perinuclear granules. The surfactant film confers alveolar stability during respiratory cycles and prevents injury to the thin extended type I epithelial cells (arrowheads).

B: Higher magnification image of a type II cell. The type II cell is the singula site of SP-C synthesis. The mature type II cell contains numerous cytoplasmic lamellar bodies (arrow) formed by concentric lipid sheets. Lamellar bodies are the mature stored surfactant secretory granules.

C: High magnification depicts maturation of the lamellar body. Pre-lamellar multivesicular body (MVB) contains small membrane bound vesicles that fuse to form the lamellar body. MVB/LB indicates a transition structure of MVB partially incorporated into the growing lamellar body (LB) via continuous outer limiting membrane and internal vesicle-to-lamellar membranes. SP-C has been localized to the MVB and LB [11].

Magnifications: A: $2,700 X$ B: $8,000 X$ C: $27,000 X$ Micrographs courtesy of Dr Cheng-Lun $\mathrm{Na}$ biosynthetic process. Pulmonary surfactant is synthesized and secreted by a distinct subset of cuboidal epithelial cells (type II cells) in the alveolus while gas exchange occurs across thin highly attenuated type I epithelial cells. Phospholipids and surfactant proteins are assembled into multivesicular bodies that coalesce into concentric membranous lamellae containing organelles. These lamellar bodies serve as the intracellular storage form of surfactant in the type II cells (Figure 1). Exocytosis of the lamellar body into the extracellular space results in a rapid unraveling and release of phospholipid rich material that ultimately reorganizes into a monolayer across the surface of the alveolus $[10,11]$. The compression and expansion of the phospholipids in the surfactant film lower surface tension and impart resistance to collapse during respiration. During repetitive breathing extracellular forms of surfactant are altered and depleted of SP-B/SP-C. Surfactant is internalized by type II cells in a recycling pathway for re-incorporation into surface-active material. Rare forms of newborn or childhood interstitial lung disease are caused by mutations that impede surfactant production and metabolism. Altered type II cell specific phospholipid routing disrupts lamellar body formation and results in a fatal surfactant dysfunction. Alveolar surfactant levels are also regulated by macrophages that phagocytose and degrade surfactant. Impaired surfactant catabolism results in a clearance disorder termed alveolar proteinosis where the alveolar lumens fill with surfactant material and alters respiratory function [12].

\section{SP-C Structure, Processing, and Function}

SP-C is expressed as a 197 amino acid proprotein of approximately $21 \mathrm{kD}$ that consists of three distinct domains: 1) a short 23 amino acid $\mathrm{N}$-terminal peptide required for late endosome routing, 2) a hydrophobic helical transmembrane domain which corresponds to the mature, secreted form of SP-C, and 3) a $14 \mathrm{kD}$ C-terminal peptide containing a BRICHOS 'chaperone' domain. The BRICHOS related region of proSP-C is a structurally similar domain found in previously unrelated rare degenerative diseases and forms of cancer $[8,13]$. After transport from the ER to the Golgi, the adjacent cysteines of the transmembrane domain are palmitoylated. This in turn initiates endocytic translocation to multivesicular bodies where sequential proteolysis steps eliminate the long C-terminal domain. Upon condensation of the multivesicular bodies into densely packed lamellar bodies, the short $\mathrm{N}$-terminal domain is proteolytically removed yielding a 35 amino acid peptide of $3.7 \mathrm{kD}$ that is the mature form of SP-C secreted into the airspace.

The central hydrophobic domain of mature SP-C is comprised of a valine rich region ( 10 of 16 residues) flanked by additional hydrophobic amino acids. This domain is configured as a trans-membrane spanning alpha helix with covalent attachment of palmitoyl groups at two adjacent amino terminal cysteine residues. Thus SP-C is a true lipopeptide able to associate with the surfactant phospholipid film, cellular membrane structures, or membrane associated proteins via the hydrophobic helical domain and/or the palmitoyl side chains. Historically, the function of SP-C has been associated with surface active properties imparted by both the helical domain and the palmitoyl chains. Insertion of SP-C alters phospholipid packing and facilitates the rapid spreading of surfactant lipids and imparts stability of surfactant monolayer films $[4,8,10]$. While SP-C does exhibit surface tension lowering properties, these do not approach the efficiency exhibited by the amphipathic SP-B to lower surface tension to near-zero $(\mathrm{mN} / \mathrm{m})$ values. However, fully palmitoylated SP-C is required for optimal surface active properties and has been further shown to exhibit synergistic surface activity with 
Citation: Glasser SW, Baatz JE, Korfhagen TR (2011) Surfactant Protein-C in the Maintenance of Lung Integrity and Function. J Aller Ther S7:001. doi:10.4172/2155-6121.S7-001

SP-B [14]. It should also be emphasized that the palmitoyl groups and adjacent amino acids have also been shown to promote binding to phosphatidylglycerol and lipopolysaccharide, the latter of which is unrelated to its surface active properties. Palmitoylation of several proteins, including $G$ proteins, facilitates interaction with membrane associated receptor proteins, offering an additional potential role for the palmitoyl groups of SP-C.

Owing to the surface active properties of SP-B and SP-C, extracts of porcine and bovine surfactant are now used clinically to rescue neonatal respiratory distress. These surfactant extracts contain variable amounts of SP-B and are enriched in SP-C [15]. Instillation of SPC-phospholipid preparations into the lungs of surfactant deficient animals improved the outcome of experimental RDS. Recombinant SP-C based preparations decreased injury in both a preterm animal model of pulmonary surfactant deficiency and animals with acute lung injury induced by lavage depletion of surfactant [16-18]. A synthetic surfactant comprised of recombinant SP-C and synthetic phospholipids has been tested in trials for adult RDS with limited efficacy [19]. The poor outcome of the clinical trials may reflect the complex origins, advanced injury, and spectrum of disorders grouped into adult RDS relative to the developmental deficiency that is newborn RDS.

As is discussed in latter sections, several lung diseases are associated with alterations in the stability of either the helical domain or acylated cysteines of mature SP-C. For example, SP-C isolated from broncho-alveolar lavage of patients with pulmonary alveolar proteinosis exists as misfolded amyloid-like fibrils, where the helical region of SP-C is transformed into beta-sheet aggregates. The beta transformation results in SP-C fibril structures that are associated with plaque induced injury [20,21]. Such structures have been recapitulated in vitro by deacylation and acidification [20]. Comparative analysis of native and deacylated SP-C indicates that acylation is required to stabilize the helical conformation of mature SP-C and increases surface phospholipid interactions [22,23]. In addition, the BRICHOS related region of proSP-C appears to function as an intramolecular chaperone that induces proper SP-C (helical) conformation of the transmembrane region (corresponding to mature SP-C) during the early stages of
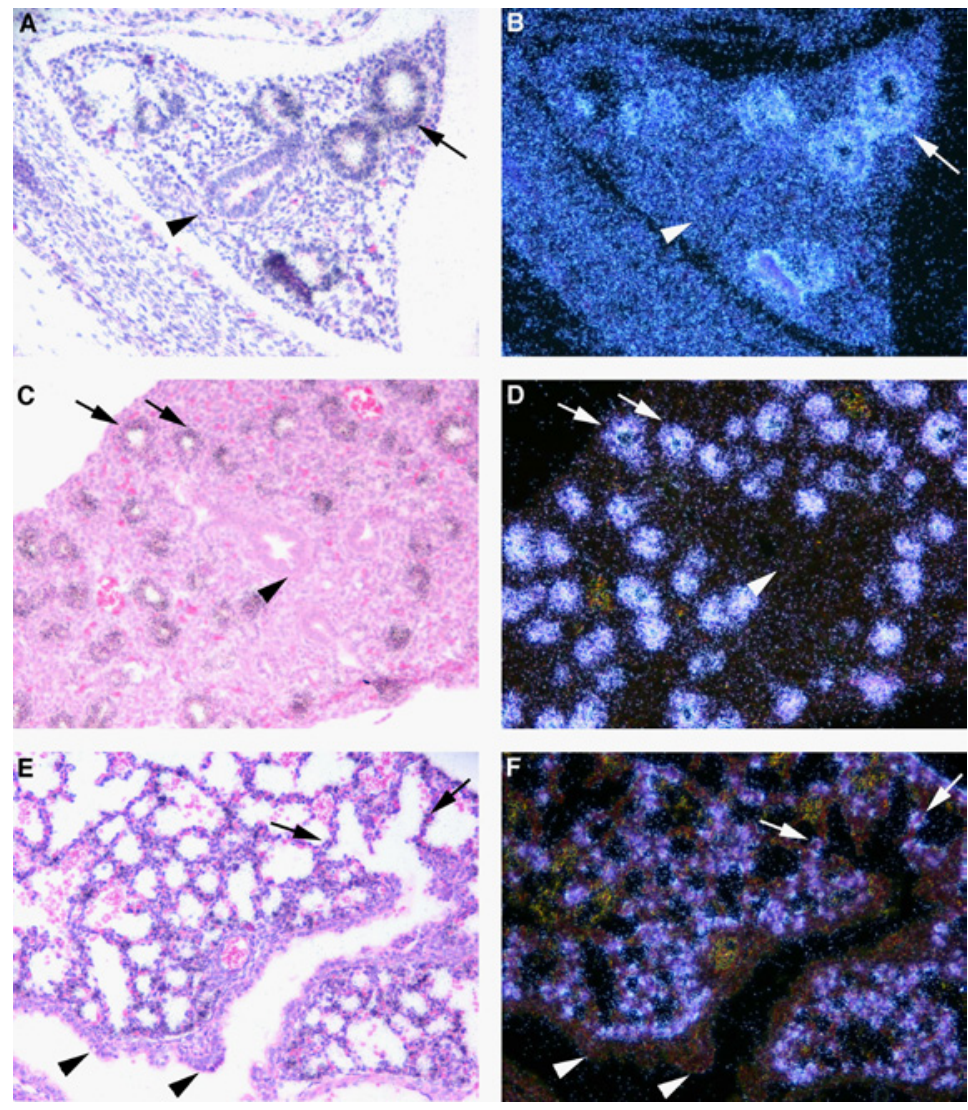

Figure 2: Pattern of SP-C expression during lung development

In situ hybridization was used to identify sites of SP-C expression in the developing mouse lung: embryonic day (ED) ED13 (panels of A, B), ED15 (panels of C, D), ED18 (panels of E, F).

A, C, E are bright field images to visualize tissue morphology.

$\mathrm{B}, \mathrm{D}, \mathrm{F}$ are corresponding dark field image where hybridization of the SP-C riboprobe to SP-C mRNA appears as light granules. Arrowheads indicate central airway epithelia, arrows indicate peripheral extension of the growing airway(s) epithelia where SP-C is expression is sustained.

Weak SP-C expression is detected in cells of the primitive airways of the ED13 lung rudiment where branch formation has begun. SP-C expression is already decreased in the central most tubule (arrowhead panels A, B).

The relative expression of SP-C has increased and localizes to the extending epithelium in ED15 lung. As branching morphogenesis proceeds SP-C expression is silenced in cells lining the larger central airways (arrowhead) and restricted to the most peripheral aspects of the invading epithelium.

By ED18 lung mesenchyme has thinned and septation has begun. SP-C expression is extinguished in the conducting airway epithelia and is only detected as strong focal sites of expression in the alveolarizing compartment. All images 20X original magnification. 
processing $[24,25]$. The BRICHOS region of proSP-C has been shown to bind to the unfolded poly-valine region and facilitate alpha helical formation of mature SP-C $[19,20]$. Genetic mutations leading to specific amino acid substitutions in the BRICHOS region of proSP-C have been associated with RDS and familial SP-C deficiencies.

\section{The pattern of SP-C expression is developmentally regulated}

Each surfactant protein has distinct patterns of expression in a subset of respiratory epithelial cells that include the alveolar type II cell. Type II cells have regenerative properties, proliferating to renew the alveolar epithelial surface following injury. SP-C is the most restricted of the surfactant proteins being detected only in the surfactant producing type II cell in the adult lung while SP-A, B, and D are expressed in tracheal gland cells (SP-A) and the bronchiolar -Clara cell epithelial population as well as alveolar type II cells. SP-C is the earliest expressed surfactant protein. It is produced at high abundance early in organogenesis [26]. In the mouse embryo the lung rudiment emerges from the endodermal foregut as distinct buds at embryonic day (ED) $9.5-10.0$. SP-C mRNA is detected as early as ED11. SP-C mRNA is readily detected by in situ hybridization in the most distal subset of cells lining the primitive airway rudiments (Figure 2A, B). SP-B expression is detected approximately 2 days later and SP-A, SP-D still later. As lung morphogenesis progresses SP-C expression is sustained in cells at the tips of peripheral elongating branches and extinguished in the cells lining the central and proximal tubules (Figure 2, C-F) [26]. The relative expression of SP-C increases throughout development most likely reflecting the increase in the number of terminal buds generated by branching morphogenesis harboring SP-C positive cells. Ultimately SP-C gene expression is detected in a focal pattern throughout the alveolar-parenchymal compartment of the adult mouse lung that is consistent with the location of differentiating type II cells.

SP-C expression is intimately linked to differentiation and maintenance of the type II cell in vivo. In a model of forced ectopic differentiation SP-C expression is induced when heterologous nonalveolar tissue is reprogramed in vitro to assume a distal parenchymal appearance. When embryonic tracheal epithelial rudiments are excised, stripped of mesenchyme, and cultured in a defined matrix-media that supports type II cell differentiation the tracheal epithelium buds and branches to form alveolar-like structures. At the ultrastructure level the cells lining the tracheal buds trans-differentiate into surfactant producing type II cells. SP-C expression is detected by in situ hybridization at the tips of distal extending airways in a pattern that matches the intrinsic SP-C expression observed during embryonic lung maturation [27]. Thus SP-C is the earliest surfactant marker of the pretype II cells during organogenesis, is the only surfactant protein gene expressed just in the alveolar type II cell and serves as the most specific marker of alveolar maturation, injury, or successful remodeling.

\section{Analysis of the SP-C promoter has identified enhancers and transcription factors that dictate cell selective gene expression}

Functionally testing regions of the human SP-C gene promoter in transgenic mice identified the segments of DNA that directed lung specific gene expression. $3.7 \mathrm{kB}$ of the human SP-C promoter proximal genomic DNA conferred lung specific expression of a linked reporter gene $[28,29]$. Transgene expression was localized to distal respiratory and alveolar epithelial cells. $4.8 \mathrm{kB}$ of the mouse SP-C gene promoter also produced strong lung specific expression in transgenic mice. In vivo deletion analysis localized an essential lung specific element to $318 \mathrm{bp}$ of DNA adjacent to the basal promoter. Lung expression from the 318bp SP-C transgenic lines was extremely weak and inferred that a strong enhancer resides in the more distal region of the $4.8 \mathrm{kB}$ of genomic DNA [30]. Cell transfection experiments confirmed the existence and nature of the distal enhancer region. In transient cell transfection assays the $4.8 \mathrm{kB}$ SP-C promoter constructs produced low-level expression while in stable cell transfection experiments the promoter activity increased by almost two orders of magnitude. An increase in expression after stable integration did not occur with the 318 bp SP-C construct. Increased SP-C driven expression following stable transfection indicates that the upstream region of the $4.8 \mathrm{kB}$ SP-C promoter construct contains an enhancer that requires chromatin integration to impart the high level SP-C expression.

The 318 bp of SP-C genomic DNA that directed in vivo lung specific expression has been used to identify transcription factors that activate SP-C expression. Cell transfection experiments with a $318 \mathrm{bp}$ SP-C promoter-luciferase reporter plasmid in combination with candidate transcription factor constructs revealed that the homeodomain factor Nkx2.1 stimulates the SP-C promoter by interaction at two adjacent binding sites [31]. Nkx2.1 is expressed in a limited set of organs including the brain, thyroid, and lung. Nkx2.1 is expressed in the earliest developing lung rudiment and loss of the Nkx2.1 expression in vivo disrupts lung morphogenesis indicating that $\mathrm{Nkx} 2.1$ is essential for lung organogenesis [32-34]. Nkx2.1 activates the SP-A and SP-B promoters as well, indicating that it has a central role in the regulation of surfactant protein gene expression. The transcription factors Gata6, NF1, TAZ, and Erm-1 stimulate SP-C expression and bind in close approximation to the Nkx2.1 sites. Similar to Nkx2.1, Gata-6 is a driver of distal lung morphogenesis and is expressed in alveolar type II cells. Over-expression of Gata- 6 or expression of dominant negative Gata-6 impaired alveologenesis in the developing lung [35,36]. Gata6, TAZ, NF1 and Erm-1 were shown to interact directly with Nkx2.1 and synergistically stimulate SP-C expression [37-40]. Collectively these findings identify a set of factors that are critical for directing lung formation and also directing SP-C gene transcription.

\section{SP-C deficiency and mutations are associated with rare familial ILD and increase susceptibility to injury}

Although uncommon some full term infants have respiratory distress at birth while others will develop respiratory diseases in later infancy and childhood. While phenotypically heterogeneous, these disease are linked to mutations that inhibit surfactant function and are collectively termed surfactant dysfunction diseases [41,42]. Mutations that alter SP-B structure or expression result in an immediate fatal RDS at birth [43]. Ultrastructure studies from infants with SP-B mutations or mice with an inactivated SP-B gene reveal common features of incomplete intracellular lamellar body formation and reduced airspace surfactant that has poor surface activity. Infants with SP-B mutations also have misprocessed proSP-C indicating that both surfactant proteins are depleted and that SP-B and SP-C processing are interdependent [44]. Individuals with familial forms of interstitial lung disease (ILD) were linked to impaired SP-C expression. The pulmonary phenotype associated with abnormalities of SP-C expression is more heterogeneous than neonatal SP-B deficiency. SP-C related respiratory decline may occur as an acute post partum crisis, emerge slowly in childhood or go undetected until later in life. The clinical profile of adult onset SP-C related lung disease varies from mild ILD to severe pulmonary fibrosis [45]. SP-C deficiency has also been attributed to the onset of bovine and canine pulmonary fibrosis $[46,47]$.

Mutations in the SP-C gene (SFTPC) are the underlying molecular lesions of SP-C dysfunction disease $[5,45]$. Mutations alter proSP-C 
structure and reduce levels of mature SP-C. The inability to correctly process aberrant forms of proSP-C can generate a cellular injury derived from misfolded protein stress responses, the accumulation of aggregated forms of pro-SP-C or from the absence of mature SPC. The majority of SFTPC mutations map to the carboxy terminal region of proSP-C that harbors the BRICHOS domain. The BRICHOS region can bind the polyvaline hydrophobic domain of SP-C and induce proper helical formation inferring the BRICHOS segment of proSP-C functions as a molecular chaperon. The predominance of the BRICHOS mutants indicates an essential role for this domain in SP-C maturation and assimilation into lamellar bodies. Several SFTPC mutations occur more frequently in affected individuals. The most common variants include single nucleotide changes that eliminate an mRNA splice site to delete exon 4 (DelEx4), substitutions of a single amino acid leucine to glutamine at position 188 (L188Q in exon 5) or isoleucine to threonine position 73 (I73T in exon 3 ) $[48,45,49]$. The "molecular consequences" of these structural alterations vary. Del Ex4 and L188Q mutants were found to localize as perinuclear endoplasmic reticulum aggregates along with increased components of a misfolded protein response. Expression of the non-BRICHOS I73T proSP-C generates abnormal vesicular structures that are misrouted to plasma membrane - endosomes and have reduced recycling of phospholipids [50]. Normally type II cells actively clear and reprocess pulmonary surfactant from the alveolar lumen. Individuals with I73T mutations have an accumulation of surfactant in the airspaces that is consistent with the observed misrouting and decreased reutilization of surfactant phospholipids.

SFTPC associated mutations are more frequently identified in familial forms of pulmonary fibrosis, 5 of 20 patients sequenced compared to 1 mutation from 135 patients sequenced and 0 of 35 patients with nonfamilial, sporadic IPF [51-53]. Gene sequencing of a cohort of 121 children with diffuse lung disease identified 18 SFTPC mutations with hereditary transmission for 9 individuals [54]. The fibrosis resulting from SP-C mutation/dysfunction provides compelling evidence that mutations that compromise the respiratory epithelium underlie idiopathic forms of pulmonary fibrosis. Additional mutations that support this premise are summarized in the final subsections.

Additional defects underlie SP-C related ILD. SP-C deficient disease has been identified in a smaller subset of individuals without any structural SFTPC mutation. One report identifies familial childhood ILD with intermittent oxygen requirements and frequent exacerbations and in another report the clinical manifestation was pulmonary fibrosis $[55,56]$. Three non-coding sequence polymorphisms (presumptive mutations) were found upstream of the SFTPC gene in infants with SP-C related respiratory distress. The introduction of these changes in SFTPC promoter constructs reduced the transcriptional activity in transient cell expression assays thus demonstrating that these variants were mutations capable of decreasing SP-C expression [57]. These studies support the concept that abnormal transcriptional regulatory elements are important causes of human lung diseases. The mutations are found at positions -2385 to -1167 bp from the basal SFTPC promoter and fall within a conserved region of the human and mouse SFTPC promoters that direct strong expression in transgenic mice [30]. These relatively uncommon forms of the SP-C dysfunction disease have decreased SP-C expression without altering proSP-C/SP-C structure that could generate a misfolded protein cellular injury. Such SP-C null mutations show that the observed clinical disease can be multifactorial arising from an SP-C deficient state or from cellular injury due to SP-C variants that impose a misfolding, aggregation, or misrouting injury.

\section{Mouse models reproduce features of SP-C related lung disease}

SP-C deficient (Sftpc-/-) mice have been generated by gene targeting techniques. The gene disruption completely ablates SP-C mRNA production and subsequent protein expression. The Sftpc-/mice serve as a model of SP-C loss-of-function without the potential injury induced by the malformed proSP-C. The surfactant from Sftpc-/mice was found to have subtle alterations in surface stability in vitro [58]. Sftpc-/- mice on a specific inbred background developed irregular lung inflammation and remodeling with features of ILD consistent with genetic influences modifying the human disease phenotype [59]. Genetic variability appears to complicate human SFTPC related disease wherein affected individuals within a family exhibit dramatic differences in the severity and time of onset even though they carry the same mutation [45]. Both the lack of SP-C and the overproduction of a proSP-C mutant predispose the animals to lung injury. Sftpc-/- mice had an increase fibrotic response and impaired recovery to challenge with the toxicant bleomycin [60]. Transgenic over-expression of a representative human SFTPC mutation deleting exon4 (DelEx4) profoundly disrupted normal lung morphogenesis and caused death. The injury was proportional to the level of DelEx4 expression and included hypoplasia, reduced branching morphogenesis, cystic terminal airspaces and reduced epithelial cell maturation [61]. The severe phenotype likely reflects the unrestricted early embryonic expression of DelEx4 conferred by the conventional 3.7kb SP-C promoter construct. To control the onset and duration of misexpressed proSP-C, transgenic mice were generated where the target gene (proSPC) is silent until specifically activated in the respiratory epithelium by administration of doxycycline. The doxycycline-induced activation of the human L188Q mutant proSP-C in the lungs of adult transgenic mice produced no observable lung pathology, inferring that this variant was not cytotoxic. However the L188Q mice were more sensitive to bleomycin induced lung fibrosis. Stimulation of endoplasmic reticulum stress by administration of tunicamycin further exacerbated the bleomycin induced L188Q fibrosis [62]. In summary the absence of mature SP-C (Sftpc-/- mice) or presence of aberrant precursors of SP-C (L188Q) can predispose the lung to fibrotic injury. This inability to resolve inflammation or injury may be a component of disease pathogenesis.

\section{SP-C confers an immunoprotective role against pulmonary pathogens}

Singular and recurrent lung infections have been reported to complicate the health of individuals with SFTPC mutation or nonmutation related disease $[45,53,54,63]$. The reports primarily identify viral related exacerbation that includes RSV, parainfluenza, and influenza, as well as less common bacterial pathogens. SP-C has been shown to bind to bacterial lipopolysaccharide (LPS) and to enhance the recognition of influenza-derived immunogenic peptides by the nasal epithelium and reduced influenza infectivity upon challenge [64,65] These observations support the hypothesis that SP-C has anti microbial and immuno-protective activity in the alveolar and upper airways. The non-alveolar functions likely reflect that in a healthy lung alveolar surfactant migrates up the airway and can act at distant epithelia.

Infection of strain-matched $S f t p c+/+$ and $-/-$ mice was used to test if the loss of SP-C influenced susceptibility to respiratory pathogens. Sftpc -/- mice had reduced clearance of the bacterial pathogen Pseudomonas aeruginosa. The influx of inflammatory cells was increased and tissue injury included interstitial thickening, septal fragmentation, and goblet cell hyperplasia. Alveolar macrophages had altered morphology and 
decreased phagocytic activity. The recovered Sftpc -/- macrophages expressed markers of alternatively activated macrophages and had increased production and secretion of matrix metaloproteinases that modify the extra cellular matrix [66]. This decreased antimicrobial response and expression of remodeling-related markers suggests that SP-C regulates the sentinel status of alveolar macrophages.

Sftpc-/-mice had an impaired response to challenge with respiratory syncytial virus (RSV). Viral clearance was decreased. Pulmonary pathology was increased in Sftpc -/- mice that included expanded alveolar interstitium, increased inflammatory cell infiltrates, and a goblet cell hyperplasia similar to the airway injury following $\mathrm{P}$. aeruginosa infection [67]. The increased viral inflammation in Sftpc-/mice was mediated by the innate immune receptor TLR3. In vitro, SP-C preparations decreased synthetic double stranded RNA activation of TLR3. The infection studies in Sftpc-/- mice compliment the clinical findings that individuals with reduced levels of SP-C or specific SFTPC mutations have compromised innate defense. SP-C blocks activation of viral innate receptors during infection and regulates the responsive status of alveolar macrophages. These studies show a direct role of SP-C in protecting the alveolus from microbial injury.

\section{Other pulmonary epithelial specific gene dysfunctions induce fibrotic lung disease}

SP-C associated ILD /IPF supports the concept that fibrotic lung disease can be initiated by epithelial cell crisis that eventually stimulates aberrant repair activity by fibroblasts. Recent population studies link defects of the pulmonary mucin MUC5B, surfactant protein A2 (SFTPA2), ELMOD2 and telomerase genes (TERC and TERT) as a cause of ILD and pulmonary fibrosis [68-71]. MUC5B is expressed in airway epithelial cells. A MUC5B promoter polymorphism was identified in affected individuals from an extensive population study of 82 enrolled families. MUC5B immunostaining was increased in the airway epithelium inferring that the polymorphism increases mucin expression and accounts for the epithelial directed onset of IPF. SP-A is expressed in alveolar and airway epithelial cells and submucosal glands. Distinct mutations within the SP-A2 carbohydrate recognition/lectinbinding domain were detected in two index families with a variable phenotype of pulmonary fibrosis or cancer. The telomerase complex maintains chromosomal integrity during cell replication to control proliferation. Type II cells in morphologically normal regions of lung adjacent to fibrotic areas were found to have reduced telomerase expression and increased cellular apoptosis suggesting that reparative type II cell proliferation is reduced in emerging fibrosis [72]. ELMOD2 is a GTPase that is selectively expressed in alveolar macrophages and type II cells and regulates TLR3 mediated viral responses indicating an overlap of mechanisms with the reduced TLR3 response in Sftpc -/- mice [73]. The mutations linked to these various human disease pedigrees adversely affect epithelial function and support the general concept that the epithelium drives ILD/fibrosis.

\section{Secondary mutations that influence SFTPC disease}

The natural course of parenchymal injury with SP-C related disease may be altered by additional mutations that impact surfactant synthesis and secretion. One candidate modifier is ABCA3. ABCA3 is a phospholipid transporter that is expressed specifically in alveolar epithelial cells and localizes to the lamellar body. Surfactant from the lungs of infants with $\mathrm{ABCA} 3$ gene mutations have altered phospholipid composition and reduced surface activity. Ultrastructure of the lungs from affected infants show malformed lamellar bodies in the type II cells consistent with $\mathrm{ABCA} 3$ as critical for phospholipid transport and incorporation [74]. ABCA3 gene targeted mice lack ABCA3 and have similar pathology with lamellar body defects and decreased surfactant biophysical activity. Unrelated infants with a history of lung disease were found to be heterozygous for both SFTPC and ABCA3. The compound $S F T P C / A B C A 3$ children had more severe lung disease than infants with just the specific SFTPC mutation [75]. A separate study of familial ILD detected subclinical interstitial changes in an SFTPC / $A B C A 3$ individual by high resolution computed tomography imaging. The early parenchymal alterations had not developed in the SFTPC only sibling supporting the interpretation that ABCA3 increases the severity of tissue destruction from the SFTPC defect [76].

A second potential modifier is the essential developmental control gene Nkx2.1. As detailed the Nkx2.1 gene is essential for both lung development and surfactant protein gene activation. Mutations that disable the Nkx2.1 gene produce a variable triad of thyroid, lung, and brain developmental deficits. Newborn respiratory failure is associated with approximately one half of the affected individuals. One recent report of fatal $\mathrm{Nkx} 2.1$ disease demonstrated a selective decrease in surfactant phospholipid and SP-C levels while the level of SP-B (also Nkx2.1 regulated) was unchanged [77]. This finding indicates that Nkx2.1 controls unknown steps in the surfactant phospholipid synthetic pathways and may impose a greater influence on SFTPC transcription than other $\mathrm{Nkx} 2.1$ responsive genes. The mechanism for this unequal loss of expression of an Nkx2.1 target gene (SP-C but not SP-B)is unknown. Perhaps other transcription factors compensate for the decreased Nkx2.1 activity to sustain SP-B expression. Although there was a clear selective loss of SP-C the pathogenic changes may be from yet-to-be defined co-regulators of Nkx2.1.

\section{Summary}

SP-C is expressed in a highly selective tissue and developmental pattern marking early presumptive distal lung epithelium and differentiated alveolar type II cells in the mature lung. Analysis of the SP-C promoter has identified genetic motifs that guide lung specific expression. Cloned SP-C promoter sequences have been used to target gene expression in the lung in order to model a host of lung disease processes. Disorders of SP-C expression may injure alveolar cells, modifying their functions leading to heterogeneous diffuse lung disease as well as pulmonary fibrosis. The molecular pathogenesis of SP-C dysfunction disease may be a composite of cellular injury generated by toxic proSP-C and alveolar injury from diminished airspace SP-C. Animal models validate the link of SP-C dysfunction with the human ILD. Infections in the SP-C null mice indicate that SP-C is immunoprotective suppressing bacterial and viral infection.

Abnormalities in SP-C dysfunction can be exacerbated by mutations in other genes. Homozygous recessive mutations for phospholipid transporter $\mathrm{ABCA} 3$ induce severe disease and even a single defective $A B C A 3$ allele increases the severity of SFTPC mutations. Mutations that ablate SP-B expression generate aberrant forms of proSP-C indicating that proper SP-B and SP-C processing are linked. ProSP-C binds and induces helical conformation of mature SP-C. Collectively these results provide evidence for dynamic protein interactions to achieve proper structure and assembly of surfactant in the type II cells. These protein interactions show that some disease processes are due to multiple steps in surfactant biosynthesis, composition or metabolism. Although the causes of many infant and childhood interstitial lung diseases remain unknown studies of surfactant proteins, in particular SP-C and other lung expressed proteins including ABCA3 and NKX 2.1 show that 
Citation: Glasser SW, Baatz JE, Korfhagen TR (2011) Surfactant Protein-C in the Maintenance of Lung Integrity and Function. J Aller Ther S7:001. doi:10.4172/2155-6121.S7-001

single or multiple gene mutations and their altered protein function in the pathogenesis of significant human lung diseases.

\section{Acknowledgements}

Work supported by NIH grant HL50046 (SWG, TRK) and HL085738 (JEB)

\section{References}

1. Thurlbeck WM (1982) Postnatal human lung growth. Thorax 37: 564-571.

2. Massaro D, Massaro GD (2007) Developmental alveologenesis: longer, differential regulation and perhaps more danger. Am J Physiol Lung Cell Mol Physiol 293: L568-L569.

3. Randell SH, Mercer RR, Young SL (1989) Postnatal growth of pulmonary acini and alveoli in normal and oxygen-exposed rats studied by serial section reconstructions. Am J Anat 186: 55-68.

4. Whitsett JA, Weaver TE (2002) Hydrophobic surfactant proteins in lung function and disease. N Engl J Med 347: 2141-2148.

5. Whitsett JA, Wert SE, Weaver TE (2010) Alveolar surfactant homeostasis and pathogenesis of pulmonary disease. Ann Rev Med 61: 105-119.

6. Wright JR (2005) Immunoregulatory functions of surfactant proteins. Nat Rev Immunol 5: 58-68.

7. Hawgood S, Derrick M, Poulain F (1998) Structures and properties of surfactant protein B. Biocim Biophys acta 1408: 150-160.

8. Beers MF, Mulugeta S (2005) Surfactant protein C biosynthesis and its emerging role in conformational lung disease. Ann Rev Physiol 67: 663-696.

9. Glasser SW, Senft AP (2011) Use of transgenic mice to understand the origins of familial pulmonary fibrosis. Current Pharm Bio 12: 1-8.

10. Perez-Gil J (2008) Structure of pulmonary surfactant membranes and films: The role of proteins and lipid-protein interactions. Biochim Biophys Acta 1778: 1676-1695.

11. Weaver TE, Na CL, Stahlman M (2002) Biogenesis of lamellar bodies, lysosome-related organelles involved in storage and secretion of pulmonary surfactant. Cell Devel Biol 13: 263-270.

12. Trapnell BC, Whitsett JA, Nakata K (2003) Pulmonary alveolar proteinosis. N Engl J Med 349: 2527-2539.

13. Willander H, Hermansson E, Johansson J, Presto J (2011) BRICHOS domain associated with lung fibrosis, dementia and cancer- a chaperone that prevents amyloid fibril formation? FEBS J 278: 3893-3904

14. Baumgart F, Ospina OL, Mingarro I, Rodriguez-Crespo I, Perez-Gil J (2010) Palmitoylation of pulmonary surfactant protein SP-C is critical for its functional cooperation with SP-B to sustain compression/expansion dynamics in cholesterol-containing surfactant films. Biophys J 99: 3234-3243.

15. Blanco O, Perez-Gil J (2007) Biochemical and pharmacological differences between preparations of exogenous natural surfactant used to treat respiratory distress syndrome: role of the different components in an efficient pulmonary surfactant. Eur J Pharm 568: 1-15.

16. Spragg RG, Smith RM, Harris K, Lewis J, Hafner D, et al. (2000) Effect of recombinant SP-C surfactant in a porcine lavage model of acute lung injury. $J$ Appl Physiol 88: 674-681.

17. Davis AJ, Jobe AH, Hafner D, Ikegami M (1998) Lung function in premature lambs and rabbits treated with recombinant SP-C surfactant. Am J Respir Crit Care Med 157: 553-559.

18. Rupert C, Kuchenbuch T, Boensch M, Schmidt S, Mathes U, et al. (2010) Dry powder arosolization of a recombinant surfactant protein -C-based surfactant for inhalative treatment of the acutely inflamed lung. Crit Care Med 38: 15841591.

19. Taut FJH, Rippin G, Schenk P, Findlay G, Wurst W, et al. (2008) A search for subgroups of patients with ARDS who may benefit from surfactant replacement therapy. Chest 134: 724-732.

20. Szyperski T, Vandenbussche G, Curtstedt T, Ruysschaert JM, Wuthrich K, et al. (1998) Pulmonary surfactant-associated polypeptide $C$ in a mixed organic solvent transforms from a monomeric alpha-helical state into insoluble betasheet aggregates. Protein Sci 7: 2533-2540.
21. Gustafsson M, THyberg J, Nasland J, Eiassson E, Johansson J (1999) Amyloid fibil formation by pulmonary surfactant protein C. FEBS Lett 464: 138-142

22. Wang Z, Gurel O, Baatz JE, Notter RH (1996) Acylation of pulmonary surfactant protein-C is required for its optimal surface active interactions with phospholipids. J Biol Chem 271: 19104-19109.

23. Dluhy RA, Shanmukh S, Leapard JB, Kruger P, Baatz JE (2003) Deacylated pulmonary surfactant protein SP-C transforms from a-helical to amyloid fibri structure via a $\mathrm{pH}$-dependent mechanism: An infared structural investigation. Biophys J 85: 2417-2429.

24. Johasson H, Nordling K, Weaver TE, Johansson J (2006) The brichos domaincontaining C-terminal part of pro-surfactant protein $\mathrm{C}$ binds to an undfolded poly-valine transmembrane segment. J Biol Chem 281: 21032-21039.

25. Johansson H, Eriksson M, Nordling K, Presto J, Johansson J (2009) The brichos domain of prosurfactant protein $\mathrm{C}$ can hold and fold a transmembrane segment. ProtSci 18: 1175-1182.

26. Wert SE, Glasser SW, Korfhagen TR, Whitsett JA (1993) Transcriptiona elements from the human SP-C gene direct expression in the primordial respiratory epithelium of transgenic mice. Dev Biol 156: 426-443.

27. Shannon JM, Gebb SA, Nielsen LD (1999) Induction of alveolar type II cell differentiation in embryonic tracheal epithelium in mesenchyme-free media. Development 126: 1675-1688.

28. Glasser SW, Korfhagen TR, Wert SE, Bruno MD, McWilliams KM, et al. (1991) Genetic elements from human surfactant protein SP-C gene confers bronchiolar-alveolar cell specificity in transgenic mice. Am J Physiol 261 L349-L356.

29. Glasser SW, Burhans MS, Eszterhas SK, Bruno MD, Korfhagen TR (2000) Human SP-C gene sequences that confer lung epithelium-specific expression in transgenic mice. Am J Physiol Lung Cell Mol Physiol 278: L933- L945.

30. Glasser SW, Eszterhas SK, Detmer EA, Maxfield MD, Korfhagen TR (2005) The murine SP-C promoter directs type II cell-specific expression in transgenic mice. Am J Physiol Lung Cell Mol Physiol 288: L625- L632.

31. Kelly SE, Bachurski CJ, Burhans MS, Glasser SW (1996) Transcription of the lung-specific surfactant protein $\mathrm{C}$ gene is mediated by thyroid transcription factor 1. J Biol Chem 271: 6881-6888.

32. Lazzaro D, Price M, de Felice M, Di Lauro R (1991) The transcription facto TTF-1 is expressed at the onset of thyroid and lung morphogenesis and in restricted regions of the foetal brain. Development 113: 1093-1104.

33. Kimura S, Hara Y, Pineau T, Fernadez-Salguero P, Fox CH, et al. (1996) The T/ebp null mouse: thyroid-specific enhancer-binding protein is essential for the organogenesis of the thyroid, lung ventral forebrain and pituitary. Genes Dev 10: $60-69$.

34. Yuan B, Li C, Kimura S, Engelhardt RT, Smith BR, et al. (2000) Inhibition of distal lung morphogenesis in Nkx2.1 (-/-) embryos. Dev Dyn 21: 180-190.

35. Liu C, Morrisey EE, Whitsett JA (2002) GATA-6 is required for maturation of the lung in late gestation.Am J Physiol Lung Cell Mol Physiol 283: L468-L475.

36. Yang H, Lu MM, Zhang L, Whitsett JA, Morrisey EE (2002) GATA-6 regulates differentiation of distal lung epithelium 129: 2233-2246.

37. Liu C, Glasser SW, Wan H, Whitsett JA (2002) GATA-6 and thyroid transcription factor-1 directly interact and regulate surfactant protein- $C$ gene expression. $J$ Biol Chem 277: 4519-4525.

38. Park KS, Whitsett JA, Di Palma T, Hong JH, Yaffe MB, et al. (2004) TAZ interacts with TTF-1 and regulates expression of surfactant protein-C. J Bio Chem 279: 17384-17390.

39. Bachurski CJ, Yang GH, Currier TA, Gronostajski RM, Hong D (2003) Nuclear factor $\mathrm{I}$ /thyroid factor 1 interactions modulate surfactant protein $\mathrm{C}$ transcription. Mol Cell Biol 23: 9014-9024.

40. Lin S, Perl AK, Shannon JM (2006) Erm/thyroid transcription factor 1 interactions modulate surfactant protein C transcription. J Biol Chem 281: 16716-16726.

41. Deterding RR (2010) Infants and young children with children's interstitial lung disease. Ped Aller Immuno Pulm 23: 25-31.

42. Wert SE, Whitsett JA, Nogee LM (2009) Genetic disorders of surfactant dysfunction. Ped Dev Pathol 12: 253-274.

43. Nogee LM, de Mello DE, Dehner LP, Colten HR (1993) Brief Report: deficiency 
Citation: Glasser SW, Baatz JE, Korfhagen TR (2011) Surfactant Protein-C in the Maintenance of Lung Integrity and Function. J Aller Ther S7:001. doi:10.4172/2155-6121.S7-001

of pulmonary surfactant protein B in congenital alveolar proteinosis. $N$ Engl $J$ Med 328: 406-410

44. Vorbroker DK, Profitt SA, Nogee LM, Whitsett JA (1995) Aberrant processing of surfactant protein $\mathrm{C}$ in hereditary SP-B deficiency. Am J Physiol Lung Cell Mol Physiol 268: L647- L656.

45. Thomas AQ, Lane K, Phillips J, Prince M, Markin C, et al. (2002) Heterozygosity for a surfactant protein $C$ gene mutation associated with usual interstitial pneumonitis and cellular nonspecific interstitial pneumonitis in one kindred. Am J Respir Crit Care Med 165: 1322-1328.

46. Danlois F, Zaltash S, Johansson J, Robertson B, Haagsman HP, et al. (2000) Very low surfactant protein $C$ contents in newborn Belgian white and blue calves with respiratory distress syndrome. Biochem J 351: 779-787.

47. Eriksson M, von Euler H, Ekman E, Nordling K, Haggstrom J, et al. (2009) Surfactant protein C in canine pulmonary fibrosis. J Vet Intern Med 23: 11701174.

48. Nogee LM, Dunbar AE, Wert SE, Askin F, Hamvas A, et al. (2001) A mutation in the surfactant protein $\mathrm{C}$ gene associated with familial interstitial lung disease. New Engl J Med 344: 573-579.

49. Brasch F, Griese M, Tredano M, Johen G, Ochs M, et al. (2004) Interstitial lung disease in a baby with a de novo mutation in the SFTPC gene. Eur Respir $J$ 24: 30-39.

50. Beers MF, Hawkins A, Maguire JA, Kotorashvili A, Zhao M, et al. (2011) A nonaggregating surfactant protein $\mathrm{C}$ mutant is misdirected to early endosomes and disrupts phospholipid recycling. Traffic 12: 1196-1210.

51. Lawson WE, Grant SW, Ambrosini V, Womble KE, Dawson EP, et al. (2004) Genetic mutations in surfactant protein $\mathrm{C}$ are a rare cause of sporadic cases of IPF. Thorax 59: 977-980.

52. Markart P, Ruppert C, Wygrecka M, Schmidt R, Korfei M, et al. (2007) Surfactant protein mutations in sporadic forms of idiopathic interstitial pneumonias. EurRespir J 29: 134-137.

53. vanMoorsel CHM, Matthijs FM, van Oosterhout FM, Barlo NP, de JongP, et al (2010) Surfactant protein C mutations are the basis of a significant portion of adult familial pulmonary fibrosis in a Dutch cohort. Am J RespirCrit Care Med 182: $1419-1425$.

54. Guillot L, Epaud R, thouvenin G, Jonard L, Mohsni A, et al. (2009) New surfactant protein $\mathrm{C}$ gene mutations associated with diffuse lung disease. $J$ Med Genet 46: 490-494.

55. Amin RS, Wert SE, Baughman RP, Tomashefski JF, Nogee LM, et al. (2001) Surfactant protein deficiency in familial interstitial lung disease. J Pediatrics 139: 85-92.

56. Tredano M, Griese M, Brasch F, Schumacher S, de Blic, et al. (2004) Mutation of SFTPC in infantile pulmonary alveolar proteinosis with or without fibrosis lung disease. Am J Med Genet A 126: 18-26.

57. Wambach JA, Yang P, Wegner DJ, An P, Hackett BP, et al. (2010) Surfactant protein-C promoter variants associated with neonatal respiratory distress syndrome reduce transcription. Pediatric Res 68: 216-220.

58. Glasser SW, Burhans MS, Korfhagen TR, Na CL, Sly PD, et al. (2001) Altered stability of pulmonary surfactant in SP-C deficient mice. Proc Natl Acad Sci 98 : 6366-6371.

59. Glasser SW, Detmer EA, Ikegami M, Na CL, Stahlman MT, et al. (2003) Pneumonitis and emphysema in SP-c gene targeted mice. J Biol Chem 278: 14291-14298.

60. Lawson WE, Polosukhin VV, Stathopoulos GT, Zoia O, Han W, et al. (2005) Increased and prolonged pulmonary fibrosis in surfactant protein-C deficient mice following intratracheal bleomycin. Am J Pathol 167: 1267-1277.

61. Bridges JP, Wert SE, Nogee LM, Weaver TE (2003) Expression of a human surfactant protein $\mathrm{C}$ mutation associated with interstitial lung disease disrupts lung development in transgenic mice. J Biol Chem 278: 52739-52746.

62. Lawson WE, Cheng DS, Degryse AL, Tanjore H, Polosukhin VV, et al. (2011) Endoplasmic reticulum stress enhances fibrotic remodeling in the lungs. Proc Natl Acad Sci 108: 10562-10567.

This article was originally published in a special issue, Pulmonary developmental biology handled by Editor(s). Dr. Rodolfo de Paula Vieira, University Hospital Freiburg, German
63. Chibbar R, Shih F, Baga M, Torlakovic E, Ramlall K, et al. (2004) Nonspecific interstitial pneumonia and usual interstitial pneumonia with mutation in surfactant protein C in familial pulmonary fibrosis. Mod Pathol 46: 973-980.

64. Augusto L, Le Blay K, Auger G, Blanot D, Chaby R (2001) Interaction of bacterial lipopolysaccharide with mouse surfactant protein $C$ inserted into lipid vesicles. Am J Physiol Lung Cell Mol Physiol 281: L776-L785.

65. Mizuno D, Kimoto T, Takei T, Fukuta A, Shinahara W, et al. (2011) Surfactant protein $\mathrm{C}$ is an essential constituent for mucosal adjuvanicity of Surfacten acting as an antigen delivery vehicle and inducing both local and systemic immunity. Vaccine 29: 5368-5378.

66. Glasser SW, Senft AP, Whitsett JA, Maxfield MD, Ross GF, et al. (2008) Macrophage dysfunction and susceptibility to pulmonary Pseudomonas aeruginosa infection in surfactant protein C-deficient mice. J Immunol 181: 621-628.

67. Glasser SW, Witt TL, Senft AP, Baatz JE, Folger D, et al. (2009) Surfactant protein $\mathrm{C}$ deficient mice are susceptible to respiratory syncytial virus infection. Am J Physiol Lung Cell Mol Physiol 297: L64- L72.

68. Seibold MA, Wise AL, Speer MC, Steele MP, Brown KK, et al. (2011) A common MUC5B promoter polymorphism and pulmonary fibrosis. $\mathrm{N}$ Engl $J$ Med 364: 1503-1512.

69. Wang Y, Kuan PJ, Xing C, Cronkhite JT, Torres F, et al. (2009) Genetic defects in surfactant protein $\mathrm{A} 2$ are associated with pulmonary fibrosis and lung cancer. Am J Human Genet 84: 52-59.

70. Hodgson U, Pullkkinen V, Dixin M, Peyrard-Janvid M, Rehn M, et al. (2006) ELMOD2 is a candidate gene for familial pulmonary fibrosis. Am $\mathrm{J}$ Human Genet 79: 149-154.

71. Calado RT, Young NS (2009) Telomerase diseases. N Engl J Med 361: 2353 2363.

72. Waisberg DR, Barbas-Filho JV, Parra ER, Fernezlian S, de Carvalho CRR, et al. (2010) Abnormal expression of telomerase/apoptosis limits type II alveola epithelial cell replication in the early remodeling of usual interstitial pneumonia/ idiopathic pulmonary fibrosis. Human Pathol 41: 385-391.

73. Pulkkinen V, Bruce S, Rintahaka J, Hodgson U, Laitinen T, et al. (2010) ELMOD2, a candidate gene for idiopathic pulmonary fibrosis, regulates antivira responses. FASEB J 24: 1167-1177.

74. Schulenin S, Nogee LM, Annilo T, Wert SE, Whitsett JA, et al. (2004) ABCA3 gene mutations in newborns with fatal surfactant deficiency. N Engl J Med 350 1296-1303.

75. Bullard JE, Nogee LM (2007) Heterozygosity for ABCA3 mutations modifies the severity of lung disease associated with a surfactant protein C gene (SFTPC) mutation. Pediatric Res 62: 176-179.

76. Crossno PF, Polosukhin VV, Blackwell TS, Johnson JE, Markin C, et al. (2010) Identification of early interstitial lung disease in an individual with genetic variation in ABCA3 and SFTPC. Chest 137: 969-973.

77. Kleinlein B, Griese M, Liebisch G, Krude H, Lohse P, et al. (2011) Fata neonatal respiratory failure in an infant with congenital hypothyroidism due to haploinsufficiency of the NKX2-1 gene: alteration of surfactant homeostasis Arch Dis Child Neonatal Ed 96: F453-F456. 